\title{
La evaluación de las virtudes y fortalezas del carácter en niños argentinos: adaptación y validación del Inventario de Virtudes y Fortalezas Para Niños (IVyF Niños) ${ }^{1}$
}

\section{The assessment of character's virtues and strengths in Argentine children: Adaptation and validation of the Inventory of Virtues and Strengths for Children (I.V\&S. Children)}

\author{
Aldana Sol Grinhauz ${ }^{2} \quad$ Alejandro Castro Solano \\ Universidad de Buenos Aires Universidad de Palermo
}

(Rec: diciembre 2013 - Acep: junio 2014)

\begin{abstract}
Resumen
Este trabajo tuvo como objetivo la adaptación y validación del Inventario de Virtudes y Fortalezas IVyF (Cosentino \& Castro Solano, 2008) en niños de 10 a 12 años. El IVyF Niños constituye un instrumento útil para evaluar las virtudes y fortalezas humanas de acuerdo a la clasificación del carácter de seis virtudes y 24 fortalezas de Peterson y Seligman (2004). Participaron de este estudio 518 niños (254 varones y 263 mujeres) que tenían entre 10 y 12 años y que asistían a escuelas públicas y privadas de la Ciudad de Buenos Aires (Argentina). Respecto de la estructura factorial del IVyF Niños, el análisis factorial exploratorio permitió extraer seis factores que explicaron el $46.15 \%$ de la varianza. El análisis factorial confirmatorio mostró un adecuado ajuste al modelo. En cuanto a evidencias de validez convergente, se encontraron correlaciones estadísticamente significativas y positivas, entre las puntuaciones arrojadas por: el IVyF Niños y la Escala de Deseabilidad Social Infantil (EDESI, Lemos, 2005), el Cuestionario Argentino de Personalidad Infantil (C.A.P.I., Lemos, 2006) y la pregunta sobre satisfacción con la vida: “¿Cuán satisfecho o contento te encuentras con tu vida?”.
\end{abstract}

Palabras claves: virtudes, fortalezas, evaluación, niños, psicología positiva.

\begin{abstract}
This study aimed the adaptation and validation of the Inventory of Virtues and Strengths (I.V.\&S., Cosentino \& Castro Solano, 2008) in children aged 10-12 years. The I.V.\&S. Children is a useful tool for assessing human virtues and strengths according to Peterson and Seligman's (2004) six virtues and 24 strengths classification of character. 518 children ( 254 males and 263 females) between 10 and 12 years and attending to public and private schools of the City of Buenos Aires (Argentina) were part of this study. Regarding the I.V.\&S. Children factor's structure, the exploratory factor analysis allowed the extraction of six factors that accounted for 46.15 $\%$ of the variance. Confirmatory factor analysis showed an adequate fit to the model. As to convergent validity evidence, statistically significant and positive correlations were found between scores by I.V\&S. Children and Children's Social Desirability Scale (EDESI, Lemos, 2005), the Argentine Children's Personality Questionnaire (ICSC, Lemos, 2006) and the question about life satisfaction: "How satisfied or happy are you with your life?".
\end{abstract}

Keywords: virtues, strengths, assessment, children, positive psychology.

\footnotetext{
Proyecto subsidiado por la Universidad de Buenos Aires. UBACyT: 2013-2016, 20020120200076BA, Virtudes y Fortalezas del carácter según las propuestas de la psicología positiva. Una aproximación ética como base para el diseño de instrumentos de evaluación psicológica. Buenos Aires, Argentina.

2 Correspondencia dirigida a: Aldana Sol Grinhauz, O’Higgins 2613 - 1ro depto “A”, Buenos Aires, Argentina.Teléfono: (+54 11) $4787-0942$. Correo electrónico: solgrin@yahoo.com.ar.
} 
La psicología positiva (PP) es la rama de la psicología que se ocupa del estudio científico de los aspectos positivos del ser humano (Seligman \& Csikszentmihalyi, 2000). Para ello, abarca tres áreas principales: la subjetiva, la individual y la institucional. En el área subjetiva se estudia las experiencias subjetivas que son valoradas positivamente, como las emociones positivas, la satisfacción con la vida y el bienestar. El área individual se ocupa de los rasgos individuales positivos tales como las virtudes y fortalezas de carácter. El área institucional comprende la práctica de los rasgos positivos, orientándose hacia un propósito que trascienda el interés personal, es decir, se encuentra orientada hacia el logro de relaciones interpersonales satisfactorias y saludables en los diferentes contextos, tales como la familia, el trabajo o la escuela (Seligman, 2002).

Si bien Park y Peterson (2009) mencionan que el buen funcionamiento en la vida es el resultado de la combinación de estas tres áreas, el área individual, que comprende el estudio de las virtudes y fortalezas del carácter, ocupa un papel central en la promoción del bienestar psicológico debido a que favorece el desarrollo de las otras dos áreas comentadas. La puesta en práctica de las fortalezas del carácter promueve la sensación subjetiva de bienestar y las emociones positivas por un lado y, por el otro, las instituciones positivas están formadas por personas de buen carácter.

Peterson y Seligman (2004) han brindado un importante aporte al estudio de las fortalezas y virtudes de carácter, desarrollando una clasificación denominada Values In Action (VIA) con el propósito de establecer un vocabulario común que oriente la investigación acerca de los rasgos de carácter, favorezca el diseño de instrumentos para evaluarlos y el desarrollo de intervenciones para promoverlos. En esta clasificación se identificaron seis virtudes presentes en la mayoría de las tradiciones filosóficas y religiosas de Oriente y Occidente: el coraje, la justicia, la humanidad, la templanza, la sabiduría y la trascendencia. A su vez, los autores asociaron cada una de estas virtudes con un número determinado de 24 fortalezas de carácter (Dahlsgaard, Peterson \& Seligman, 2005). En la tabla 1 se presenta la clasificación de las seis virtudes y 24 fortalezas propuesta por Peterson y Seligman (2004).

Tabla 1

Clasificación de las seis virtudes y 24 fortalezas (Peterson \& Seligman, 2004).

\begin{tabular}{ll}
\hline Virtudes & Fortalezas \\
\hline Coraje & Valentía, Persistencia, Integridad y Vitalidad \\
Justicia & Ciudadanía, Liderazgo e Imparcialidad \\
Humanidad & Amor, Bondad, Inteligencia social \\
Sabiduría & Perspectiva, Apertura mental, Amor por el saber, Curiosidad \\
& y Creatividad \\
Templanza & Clemencia, Humildad, Prudencia y Autorregulación \\
Trascendencia & Apreciación por la belleza, Gratitud, Esperanza, Humor y \\
& Espiritualidad \\
\hline
\end{tabular}

A partir del establecimiento de la clasificación VIA (Peterson \& Seligman, 2004) se han desarrollado varios estudios que produjeron diferentes tipos de evidencia empírica en relación a las fortalezas del carácter. No obstante, la mayoría de estos estudios se realizaron con población adulta mientras que muy pocas investigaciones se realizaron con niños.

A pesar de ello, los principales exponentes de la PP consideran que las fortalezas del carácter pueden ser enseñadas y adquiridas con la práctica, constituyendo la infancia, uno de los momentos más oportunos para hacerlo. Esto se debe a que durante las transiciones de desarrollo, el niño se somete a una importante reorganización e integración de las capacidades de adaptación, de forma que, reconocer las fortalezas con las que cuenta en su carácter, le brindará una base que le permitirá afrontar los retos más importantes de esta etapa (Lampropoulos, 2001; Terjesen, Jacofsky, Froh \& DiGiuseppe, 2004).

Se ha podido constatar que, por ejemplo, la perseverancia, la apertura mental y la curiosidad, influencian positivamente el logro académico y se constituyen como fuertes predictoras del éxito escolar (Cillessen \& Rose, 2005; Kashdan \& Yuen, 2007). A su vez, el amor junto con la esperanza y la vitalidad, son las fortalezas que más se asociaron con mayores niveles 
de felicidad en niños de 3 a 9 años (Park \& Peterson 2006a). La autorregulación, la prudencia y la clemencia están relacionadas con un desarrollo psicológico positivo y una mejor adaptación en las escuelas (Cillessen \& Rose, 2005), la esperanza ha sido reconocida en diferentes estudios como protectora ante la adversidad y el estrés (Seligman, Reivich, Jaycox \& Gillham, 1995). En relación al humor, Erickson y Feldstein (2007), encontraron que los niños con buen sentido del humor tienden a ser más asertivos y competentes en contextos sociales y académicos. La gratitud por su parte, se encuentra asociada positivamente con varios indicadores saludables, tales como el afecto positivo, la satisfacción con la vida, el optimismo, el apoyo social y la conducta prosocial (Froh, Yurkewicz \& Kashdan, 2008). En términos generales, se ha podido evidenciar el importante aporte que brinda la práctica cotidiana de las fortalezas del carácter por parte de jóvenes en proceso de recuperación de enfermedades físicas y trastornos psicológicos (Peterson, Park \& Seligman, 2005); así como en la prevención de problemas de comportamiento, tales como la agresividad, la adicción al consumo de sustancias y los trastornos depresivos (Park, 2004).

Paralelamente al desarrollo de la clasificación VIA, Peterson y Seligman (2004), en colaboración con otros autores, han creado instrumentos de medida para evaluar las fortalezas del carácter.

Dentro de los instrumentos dirigidos a población adulta se puede mencionar el Values in Action Inventory of Strenghts (VIA-IS; Peterson \& Seligman, 2004), el cual consta de 240 ítems y utiliza una escala Likert de cinco alternativas. Los autores han dado cuenta de sus adecuadas propiedades psicométricas con una fiabilidad, en todas las subescalas y correlaciones test re test (con un intervalo de 4 meses), superior a .70 (Peterson \& Seligman, 2004). Sin embargo, los autores consideran que este cuestionario resulta demasiado extenso, por lo que no han descartado versiones más reducidas con el objetivo de hacerlo más accesible y atractivo para responder (Peterson \& Seligman, 2004).

En respuesta a esto, en Argentina, Cosentino y Castro Solano (2008) diseñaron el Inventario de Virtudes y Fortalezas (IVyF), un instrumento mucho más corto y práctico de administrar. El IVyF posee 24 ítems, donde cada reactivo evalúa una de las 24 fortalezas de acuerdo a la clasificación de Peterson y Seligman (2004). Este cuestionario presenta una adecuada estabilidad de las puntuaciones en el tiempo, con alfas de Cronbach superiores a .70 (Cosentino, 2009).
La presencia de este instrumento en nuestro país, ha permitido obtener información acerca de las diferentes fortalezas y grados en que se presentan en una persona, de una forma más rápida y sencilla.

Respecto de los instrumentos dirigidos a evaluar las fortalezas presentes en población infantojuvenil, debe decirse que, a pesar de los beneficios que trae aparejado la práctica cotidiana de las fortalezas en esta franja etaria, son pocos los instrumentos destinados a evaluarlas.

Dentro de estos instrumentos puede mencionarse el Values in Action Inventory of Strenghts for Youth (VIAY; Park \& Peterson, 2003; 2006b), dirigido a jóvenes entre 10 y 17 años. Este inventario consta de 198 ítems y posee adecuadas propiedades psicométricas con alfas superiores a .65 en cada escala.

También se encuentra el Valores en niños (Park \& Peterson, 2006a), destinado a evaluar las fortalezas en infantes a partir de las descripciones libres que hacen los padres sobre las características y cualidades de sus hijos.

En Argentina, se puede mencionar la Entrevista Estructurada para Evaluar Fortalezas en Niños y Adolescentes (EFNA, Mikulic \& Fernández, 2006). Esta entrevista está destinada a evaluar las fortalezas presentes en niños y adolescentes. Consta de 40 ítems, con un formato de respuesta dicotómica, que se distribuyen en seis áreas principales: Factores personales, Factores interpersonales, Fortalezas en familia de origen, Fortalezas en amigos y grupo de a pares, Fortalezas en el contexto escolar y Fortalezas en el contexto laboral.

Asimismo, se puede mencionar el Inventario de Calidad de Vida para niños y adolescentes (ICVINFANTO JUVENIL; Mikulic \& Fernández, 2006). Este inventario consiste en la evaluación de la satisfacción e insatisfacción reportada en 18 dominios vitales del niño o adolescente, los cuales incluyen por ejemplo: salud, autoestima, religión, recreación, estudio, creatividad.

Como se pudo advertir anteriormente, Argentina cuenta con investigadores dedicados a diseñar instrumentos para evaluar las fortalezas del carácter. Sin embargo, no existe en nuestro país, un instrumento que permita evaluar las 24 fortalezas presentes en la clasificación VIA en niños. Contar con un instrumento de estas características, ayudará al reconocimiento y posterior análisis de las fortalezas del carácter en los niños. Al mismo tiempo, brindará información valiosa para el diseño de programas de promoción y potenciación de estos puntos fuertes. 
En función de lo comentado, el objetivo de este estudio consiste en la adaptación y validación del Inventario de Virtudes y Fortalezas (IVyF) en niños de 10 a 12 años.

\section{Método}

\section{Participantes}

En el presente estudio se seleccionó una muestra de conveniencia compuesta por 518 niños y niñas entre 10 y 12 años que asistían a escuelas tanto públicas como privadas de la Ciudad de Buenos Aires (Argentina). La muestra se balaceó por sexo, contando con 254 varones (49.1\%) y 263 mujeres $(50.9 \%)$, de los cuales 159 tenían 10 años (30.8\%), 145 tenían 11 años (28\%) y 213 tenían 12 años (41.2\%). En cuanto a los padres, el $98 \%$ trabajaban y en su mayoría eran profesionales (47\%), pequeños comerciantes $(21 \%)$ o empleados (17\%), contando en un $70 \%$ con los estudios secundarios completos. Por lo tanto, se trató de una muestra que, en su mayoría, pertenecía a la clase socioeconómica media. Por último, se solicitó la colaboración voluntaria, anónima y no remunerada de los entrevistados.

\section{Instrumentos de recolección de datos}

Encuesta sociodemográfica: Esta encuesta relevó las variables: sexo, edad y nivel académico y ocupacional de los padres de los participantes.

Inventario de Virtudes y Fortalezas Para Niños (IVyF Niños): Este instrumento está basado en el Inventario de Virtudes y Fortalezas dirigido a población adulta (IVyF; Cosentino \& Castro Solano, 2008). El IVyF Niños es un inventario de papel y lápiz compuesto por 24 ítems de autopuntuación global directa, con un formato de respuesta tipo Likert de cinco categorías, las cuales van desde el "muy parecido a mí" a "nada parecido a mí". Cada uno de los reactivos evalúa una de las 24 fortalezas correspondientes a la clasificación VIA de Peterson y Seligman (2004).A continuación se expone un reactivo del IVyF Niños que corresponde a la Fortaleza Persistencia: "Cuando estudio, no me distraigo ni dejo mis tareas sin terminar. Aunque sea muy difícil lo que estoy haciendo y aunque ya no tenga más ganas de hacerlo, no abandono la tarea y continúo hasta el final".
Pregunta sobre Satisfacción con la Vida. Se les preguntó a los participantes "¿Cuán contento/a o satisfecho/a estás con tu vida?”, pregunta a la cual los evaluados debieron responder con un formato de respuesta tipo Likert de cinco puntos, que iba desde el "no estoy contento" hasta "estoy muy contento o satisfecho con mi vida".

Escala de Deseabilidad Social Infantil (EDESI; Lemos, 2005). Ésta permite evaluar la deseabilidad social en niños de 10 a 12 años. Fue diseñada con ocho ítems que consisten en afirmaciones, en las cuales el niño debe responder de forma dicotómica ( "sí" o " $n o$ "). Estos ocho reactivos se reparten en dos factores. En el Factor 1 se saturan los cuatro ítems relativos a los aspectos positivos o esperables desde el punto de vista de la deseabilidad social, por ejemplo: "Siempre escucho con atención cuando alguien me habla". En el Factor 2, por su parte, pesaron los cuatro ítems que reflejan los aspectos negativos o socialmente menos esperables, por ejemplo: "Alguna vez me hice como el que entendía algo, cuando en realidad no entendía nada”. Se trata de una escala que presenta buenas propiedades psicométricas con un alfa de Cronbach de .70 (Lemos, 2005).

Cuestionario Argentino de Personalidad Infantil (CAPI; Lemos, 2006). Este instrumento consiste en una adaptación del Cuestionario NEOPI-R (Costa \& McCrae, 1980; McCrae \& Costa, 1991; McCrae et al. 2004; McCrae et al., 2005; Costa, McCrae \& Martin, 2008) que operacionaliza los cinco grandes factores de la personalidad. El CAPI está dirigido a niños de 9 a 12 años, quienes deben responder a los reactivos con "sí", "no" o "algunas veces". El análisis factorial exploratorio arrojó 5 factores que explicaron el $54.88 \%$ de la varianza: Neuroticismo $(\alpha=.79)$, Extraversión $(\alpha=$ $.80)$, Apertura a la Experiencia $(a=.56)$, Conciencia ( $a$ $=.71)$ y Amabilidad $(\alpha=.77)$. A su vez, estos 5 factores contienen 14 facetas de la personalidad (Lemos, 2004). El factor Escrupulosidad contiene las facetas de orden, organización y responsabilidad. El factor Neuroticismo está integrado por las facetas de autocrítica, vulnerabilidad, ansiedad, y en sentido inverso la competencia. El factor Extraversión está integrado por una faceta que atañe a las cuestiones relacionadas con el gregarismo y las emociones positivas. Otra de las facetas que integra este factor es la confianza. En cuanto al factor Amabilidad, la autora decidió nominarlo Mesura vs. Desmesura y contiene la faceta adaptabilidad en contraposición a las facetas de actividad y búsqueda de excitación. Por último, el factor Apertura contiene las facetas de innovación y acción. 


\section{Procedimiento}

Con el objetivo de aportar evidencias de validez de contenido al IVyF Niños, el contenido de los reactivos fue sometido al juicio de tres expertos, quienes examinaron su coherencia a la luz de la teoría y la franja etaria a la cual estaban dirigidos. Se conservaron solamente aquellos ítems aprobados por la totalidad de los jueces expertos.

Posteriormente, se realizó una prueba piloto con 20 niños escolarizados de 10, 11 y 12 años. Dicha prueba fue pensada con el objetivo de evaluar criterios de claridad, habitualidad y comprensión en las expresiones utilizadas (Anastasi \& Urbina, 1998).

A estos niños se les solicitó que primeramente, respondiesen el instrumento de forma individual. Una vez respondidos los ítems, se les pidió que señalasen en una sencilla encuesta, elaborada para este fin, aquello que afectaba la comprensión de la tarea que debían realizar. Es decir, si habían encontrado términos complejos, redacción confusa y, a su vez, comprobar la adecuación del formato de respuesta utilizado.

Atendiendo a la sugerencia de los participantes, se modificaron algunas palabras, se acortaron algunos ítems y se introdujo en la escala Likert una opción neutral ("ni parecido", "ni diferente").

Una vez finalizado este proceso, los instrumentos que integraban la batería final de evaluación se administraron en forma grupal en las escuelas, con un máximo de 30 personas en cada grupo. La participación de los niños (tanto en la prueba piloto como en la administración propiamente dicha) estuvo sujeta a su consentimiento y a la previa autorización de sus padres y la institución académica. Tanto los niños como los padres y las autoridades de la institución escolar fueron informados del objetivo de la investigación, garantizando la confidencialidad de los datos obtenidos.

El total de la actividad tuvo una duración de 45 minutos. El análisis de los datos se efectuó con el procesador SPSS versión 19.0 y con el programa AMOS versión 18.

\section{Resultados}

\section{Evidencias de validez factorial de IVyF Niños}

Teniendo como meta evaluar la validez factorial de la prueba, se efectuaron análisis factoriales exploratorios utilizando el método de análisis de componentes principales con rotación tipo oblicua. En primer lugar, se verificó que la matriz de datos no fuera una matriz identidad (Test de Barlett $=\mathrm{p}<.001)$ y que pudiera resistir este tipo de análisis $(\mathrm{KMO}=.854)$. Se obtuvo una solución factorial que contemplaba seis factores diferenciados entre sí, cuyos autovalores eran mayores a uno y explicaban el $46.15 \%$ de la variancia. El factor 1 explicó el $19.71 \%$, el factor 2 el $7.44 \%$, el factor 3 el $5.40 \%$, el factor 4 el $4.64 \%$, el factor 5 el $4.61 \%$ y el factor 6 el $4.35 \%$. En la Tabla 2, se muestran los seis factores que se extrajeron.

Tabla 2

Matriz de los seis componentes rotados del Inventario de Virtudes y Fortalezas Para Niños

\begin{tabular}{lrrrrrr}
\hline Variable & 1 & 2 & 3 & 4 & 5 & 6 \\
\hline Persistencia & .71 & & & & & \\
Autorregulación & .73 & & & & \\
Apertura mental & .35 & & & & \\
Integridad & .44 & & & & \\
Prudencia & .48 & & & & \\
Humildad & .40 & & & & \\
Apreciación & & .47 & & & \\
Amor & & .64 & & & \\
Gratitud & & .61 & & & \\
Bondad & & .46 & & & \\
Perspectiva & & .35 & & & \\
Humor & & .39 & & & \\
\hline
\end{tabular}

Continuación Tabla 2. 


\begin{tabular}{|c|c|c|c|c|c|c|}
\hline Variable & 1 & 2 & 3 & 4 & 5 & 6 \\
\hline Creatividad & & & .54 & -.44 & & \\
\hline Amor por el saber & & & .69 & & & \\
\hline Inteligencia social & & & & .40 & & \\
\hline Espiritualidad & & & & .57 & & \\
\hline Valentía & & & & -.59 & & \\
\hline Imparcialidad & & & & & .43 & \\
\hline Ciudadanía & & & & & .77 & \\
\hline Liderazgo & & & & & .51 & \\
\hline Clemencia & & & & & & -.47 \\
\hline Curiosidad & & & & & & -.54 \\
\hline Esperanza & & & & & & -.68 \\
\hline Vitalidad & & & & & & -.40 \\
\hline Varianza explicada* & 19.71 & 7.44 & 5.40 & 4.64 & 4.61 & 4.35 \\
\hline
\end{tabular}

Nota. Se muestran los coeficientes mayores a .30 .

* La varianza explicada se expresa en porcentaje.

El primer factor está compuesto por las Fortalezas del Temperamento (persistencia, autorregulación, apertura mental, integridad, prudencia y humildad). El segundo factor está conformado por Fortalezas de la Empatía (apreciación de la belleza, amor, gratitud, bondad, humor y perspectiva). El tercer factor está integrado por las Fortalezas del Conocimiento (creatividad y amor por el saber). El cuarto factor está integrado por las Fortalezas Sociales (inteligencia social, espiritualidad y valentía). El quinto factor se encuentra compuesto por las Fortalezas de la Justicia (imparcialidad, ciudadanía y liderazgo). Finalmente, al sexto factor consta de Otras Fortalezas Asociadas (clemencia, curiosidad, esperanza y vitalidad).

Evidencias de validez convergente del IVyF Niños con la satisfacción con la vida y la deseabilidad social

Para aportar evidencias de validez convergente al IVyF Niños, se procedió a la realización de correlaciones bivariadas mediante el coeficiente $r$ de Pearson entre las puntuaciones de las fortalezas de carácter medidas con el IVyF Niños y las puntuaciones de la satisfacción con la vida y la deseabilidad social, medidas a partir de la pregunta "¿Cuán satisfecho/a o contento/a estás con tu vida?" y la escala EDESI, respectivamente. En la tabla 2 se presentan las correlaciones halladas.

Tabla 2

Correlaciones entre las fortalezas del carácter, la satisfacción con la vida y la deseabilidad social

\begin{tabular}{lcccc}
\hline Fortalezas & $\begin{array}{c}\text { Media } \\
\text { (desvío) }\end{array}$ & \multicolumn{2}{c}{ Satisfacción con la vida } & $\begin{array}{c}\text { Deseabilidad } \\
\text { social }\end{array}$ \\
\hline Gratitud & & $\begin{array}{c}\text { Correlaciones } \\
\text { simples }\end{array}$ & $\begin{array}{c}\text { Controlando por } \\
\text { sexo y edad }\end{array}$ \\
Bondad & $4.64(.61)$ & $.15^{* *}$ & $.14^{* *}$ & $.26^{* *}$ \\
Apreciación & $4.55(.73)$ & $.16^{* *}$ & $.13^{* *}$ & .08 \\
Vitalidad & $4.36(.78)$ & $.23^{* *}$ & $.22^{* *}$ & $.23^{* *}$ \\
Humor & $4.27(.92)$ & $.25^{* *}$ & $.24^{* *}$ & $.20^{* *}$ \\
Amor & $4.25(1.02)$ & $.14^{* *}$ & $.14^{* *}$ & .00 \\
Ciudadanía & $4.21(1.07)$ & $.18^{* *}$ & $.15^{* *}$ & .10 \\
Integridad & $4.19(.87)$ & $.13^{*}$ & $.11^{*}$ & .08 \\
\hline & $4.18(.95)$ & $.15^{* *}$ & $.13^{* *}$ & $.38^{* *}$ \\
\hline
\end{tabular}




\begin{tabular}{lrrrr}
\hline Liderazgo & $4.11(.99)$ & $.15^{* *}$ & $.14^{* *}$ & .12 \\
Espiritualidad & $4.10(1.30)$ & $.18^{* *}$ & $.16^{* *}$ & .14 \\
Curiosidad & $4.01(1.02)$ & $.21^{* *}$ & $.19^{* *}$ & $.18^{*}$ \\
Inteligencia social & $3.99(1.21)$ & $.16^{* *}$ & $.16^{* *}$ & .14 \\
Imparcialidad & $3.97(1.02)$ & $.11^{*}$ & .09 & $.23^{* *}$ \\
Creatividad & $3.90(1.16)$ & -.02 & -.04 & $.18^{*}$ \\
Apertura mental & $3.88(1.09)$ & $.10^{*}$ & .08 & $.23^{* *}$ \\
Prudencia & $3.88(1.11)$ & $.20^{* *}$ & $.17^{* *}$ & $.29^{* *}$ \\
Esperanza & $3.86(1.15)$ & $.23^{* *}$ & $.22^{* *}$ & $.23^{* *}$ \\
Valentía & $3.84(1.17)$ & -.06 & -.06 & .03 \\
Humildad & $3.79(1.24)$ & $.15^{* *}$ & $.14^{* *}$ & .05 \\
Persistencia & $3.67(1.23)$ & $.14^{* *}$ & $.13^{* *}$ & $.24^{* *}$ \\
Perspectiva & $3.51(1.16)$ & .03 & .01 & .06 \\
Autorregulación & $3.39(1.29)$ & $.18^{* *}$ & $.16^{* *}$ & $.28^{* *}$ \\
Clemencia & $3.21(1.26)$ & $.15^{* *}$ & $.12^{* *}$ & $.37^{* *}$ \\
Amor por el saber & $2.81(1.36)$ & .09 & .07 & $.21^{* *}$ \\
\hline
\end{tabular}

Nota. Se destacan en negrita las correlaciones con tamaños del efecto superiores a mediano ( $r=.30$; Cohen, 1992). Apreciación $=$ apreciación de la belleza; Apertura $=$ apertura mental.

${ }^{*} \mathrm{p}<.05$, bilateral. $* * \mathrm{p}<.01$ bilateral.

De acuerdo a la tabla anteriormente expuesta, se puede observar que se encontraron correlaciones estadísticamente significativas y directas entre la pregunta "¿Cuan satisfecho/a o contento/a te encontrás con tu vida?" y todas las fortalezas de carácter exceptuando creatividad, valentía y perspectiva. En la mayoría de los casos, estas relaciones se mantuvieron significativas al realizar un control con análisis de covarianza (ANCOVA) por las variables sexo y edad, exceptuando las fortalezas apertura, imparcialidad y amor por el saber. Esto quiere decir que, los niños que señalaron estar más satisfechos con su vida, también se autopercibieron más abiertos a explorar y descubrir nuevos temas de interés, con energía para emprender aventuras, cautos a la hora de tomar decisiones o de aconsejar a alguien, con la cualidad de poder percibir la belleza de las cosas simples de la vida y con expectativas positivas acerca del futuro.

Respecto de la deseabilidad social, los resultados mostraron que de las 24 fortalezas, 14 se asociaron de forma estadísticamente significativa con este constructo. Las fortalezas que obtuvieron las más elevadas correlaciones con deseabilidad social fueron integridad y clemencia, con un tamaño del efecto moderado. Esto significa que aquellos niños que quisieron dar una imagen positiva de sí mismos, también se autopercibieron capaces de perdonar y fieles a sus valores.

\section{Evidencias de validez convergente del IVyF Niños con la personalidad}

Se hipotetizó la existencia de correlaciones positivas entre: el factor Apertura a la Experiencia y las fortalezas apreciación de la belleza, curiosidad y creatividad; el factor Escrupulosidad y las fortalezas persistencia y autorregulación; el factor Mesura y las fortalezas bondad y gratitud y el factor Extraversión y las fortalezas vitalidad y amor (Peterson \& Seligman, 2004). Para corroborar estas asociaciones se establecieron correlaciones, mediante $\mathrm{r}$ de Pearson, entre las puntuaciones arrojadas por el IVyF Niños y las arrojadas por el CAPI (ver Tabla 3). El análisis de las correlaciones demuestra que el factor Apertura a la Experiencia presentó asociaciones bajas con dos de las fortalezas hipotetizadas: apreciación de la belleza y curiosidad. También se encontraron correlaciones bajas entre este factor y la fortaleza perspectiva. Es decir que, aquellos niños que gustan de vivir situaciones desconocidas y novedosas, también registraron en su carácter la habilidad para apreciar las cosas sencillas de la vida, conocer y aprender cosas nuevas.

A su vez, el factor Escrupulosidad registró correlaciones moderadas y significativas con las dos fortalezas hipotetizadas: persistencia y autorregulación y con las fortalezas clemencia, curiosidad, prudencia y amor 
por el saber. También presentó correlaciones bajas con imparcialidad, gratitud, liderazgo, apertura mental, inteligencia social, integridad y vitalidad. Esto significa que, los niños con una personalidad más organizada y responsable se reconocieron en mayor medida como capaces para regular sus impulsos, cautos a la hora de tomar decisiones, con una mayor afabilidad por la adquisición de conocimientos nuevos, más persistentes en sus objetivos, misericordiosos y abiertos a explorar y conocer cosas nuevas.

El factor Mesura no registró correlaciones con las dos fortalezas hipotetizadas. Sin embargo, se hallaron correlaciones moderadamente significativas con las fortalezas clemencia, prudencia y autorregulación y asociaciones bajas con las fortalezas imparcialidad, persistencia, apertura a la experiencia, esperanza, integridad y amor por el saber. Esto quiere decir que, los niños con un temperamento más mesurado se reportaron por sobre todas las cosas como prudentes, misericordiosos y capaces de regular las propias respuestas frente a los estímulos internos y externos.

Finalmente, el factor Extraversión no presentó ninguna de las correlaciones hipotetizadas. No obstante, registró asociaciones bajas con las fortalezas apreciación de la belleza, inteligencia social, bondad y esperanza. Esto implica que, los niños con una personalidad más sociable y dinámica, se reconocen como más atentos a las cosas lindas de la vida, más inteligentes socialmente, con una visión positiva del futuro y dispuestos a ayudar a quien lo necesite.

Tabla 3

Correlaciones entre las fortalezas del carácter y la personalidad

\begin{tabular}{|c|c|c|c|c|c|}
\hline Fortalezas & Neuroticismo & Extraversión & Mesura & Escrupulosidad & Apertura \\
\hline Apreciación & -.06 & $.23 * *$ & .13 & $.20 *$ & $.22 * *$ \\
\hline Imparcialidad & .01 & .12 & $.25^{* *}$ & $.22 * *$ & .02 \\
\hline Persistencia & -.12 & $.16^{*}$ & $.25 * *$ & $.37 * *$ & .03 \\
\hline Creatividad & .02 & $.20 *$ & -.04 & .15 & .15 \\
\hline Amor & $.22 * *$ & .10 & $.18^{*}$ & .16 & $.20^{*}$ \\
\hline Autorreg. & -.14 & .03 & $.33 * *$ & $.47 * *$ & .11 \\
\hline Gratitud & -.11 & .07 & .13 & $.30 * *$ & .10 \\
\hline Liderazgo & -.11 & .09 & .04 & $.26^{* *}$ & .12 \\
\hline Apertura & -.01 & .03 & $.23 * *$ & $.25^{* *}$ & .14 \\
\hline Inteligencia Social & -.06 & $.26 * *$ & .09 & $.21 * *$ & .10 \\
\hline Clemencia & -.03 & .15 & $.48 * *$ & $.34 * *$ & .08 \\
\hline Espiritualidad & .08 & .05 & .15 & .13 & $.17 *$ \\
\hline Ciudadanía & -.11 & $.16^{*}$ & .08 & $.19 *$ & .10 \\
\hline Valentía & .03 & .11 & .06 & .04 & .15 \\
\hline Curiosidad & -.01 & .08 & .11 & $.32 * *$ & $.23 * *$ \\
\hline Bondad & -.06 & $.26 * *$ & .10 & $.17 *$ & .14 \\
\hline Esperanza & -.01 & $.22 * *$ & $.21 * *$ & .14 & .04 \\
\hline Integridad & .00 & .13 & $.28 * *$ & $.25^{* *}$ & .11 \\
\hline Perspectiva & .01 & $.16^{*}$ & -.04 & .06 & $.21 * *$ \\
\hline Prudencia & .04 & $.19 *$ & $.47 * *$ & $.42 * *$ & .06 \\
\hline Humor & -.01 & .14 & -.16 & -.01 & .08 \\
\hline Humildad & .00 & .00 & .12 & $.17 *$ & $.19^{*}$ \\
\hline Amor por el saber & .06 & .05 & $.28 * *$ & $.40 * *$ & .15 \\
\hline Vitalidad & -.13 & $.17 *$ & .15 & $.27 * *$ & $.16^{*}$ \\
\hline
\end{tabular}

Nota. Se destacan en negrita las correlaciones con tamaños del efecto superiores a mediano (r=.30; Cohen, 1992). Apreciación = apreciación de la belleza; Autorreg. = Autorregulación; Apertura = apertura mental. 


\section{Diferencias en las puntuaciones del IV y $F$ Niños de acuerdo al sexo}

Para contrastar las medias de las puntuaciones del IVyF Niños de acuerdo al sexo, se realizó un análisis multivariante de la varianza (MANOVA; Peña, 2002) que incluyó al sexo como factor y las 24 fortalezas de carácter como variables dependientes.

En cuanto a las diferencias encontradas, los resultados indican la existencia de diferencias significativas entre los vectores medios de ambos grupos $\left(T^{2}=0.33\right.$, $F(24,492)=6.93, p<.001)$. Para determinar en cuáles fortalezas diferían los varones de las mujeres, se realizaron posteriormente pruebas univariadas (Peña, 2002). Los resultados indicaron que las mujeres puntuaron más alto que los varones en 12 de las 24 fortalezas del carácter. Las mayores diferencias se encontraron en las fortalezas amor $\left(M_{\text {mujeres }}=4.55, D E=.74 ; M_{\text {varones }}=\right.$ $3.85, D E=1.23, F(1,515)=60.47 ; p<.001)$, bondad $\left(M_{\text {muieres }}=4.75, D E=.50 ; M_{\text {varones }}=4.35, D E=.85\right.$, $F(1,515)=42.08 ; p<.001)$ y perspectiva $\left(M_{\text {mujeres }}=\right.$ $3.75, D E=1.05 ; M_{\text {varones }}=3.27, D E=1.21, F(1,515)$ $=22.92 ; p<.001)$.

\section{Discusión}

El objetivo de la presente investigación consistió en la adaptación y validación del Inventario de Virtudes y Fortalezas (IVyF; Cosentino \& Castro Solano, 2008) en niños de 10 a 12 años.

Respecto de las propiedades psicométricas del instrumento, se destaca primeramente que, la finalidad de haber llevado a cabo un análisis factorial exploratorio sobre las puntuaciones del IVyF Niños, no tuvo por objetivo hacer afirmaciones sobre la estructura de las fortalezas más acertada, sino mostrar coincidencias y diferencias con las estructuras factoriales obtenidas por otras investigaciones que utilizaron otros instrumentos de medición de la clasificación Values In Action.

Tras la realización del análisis factorial exploratorio, se extrajeron seis factores que explicaron el $46.15 \%$ de la varianza. El primer factor recibió el nombre de Fortalezas del Temperamento (integrado por las fortalezas prudencia, autocontrol, integridad, perseverancia, apertura Mental y humildad); el segundo factor se llamó Fortalezas de la Empatía (donde se encuentran la bondad, el amor, la gratitud, la apreciación de la belleza, la perspectiva y el humor); el tercer factor se denominó Fortalezas Intelectuales (integrado por las fortalezas creatividad y amor por el conocimiento); el cuarto factor se designó como Fortalezas Sociales (compuesto por la inteligencia social, la espiritualidad y la valentía); el quinto factor se nombró Fortalezas de la Justicia (conformado por las fortalezas ciudadanía, imparcialidad y liderazgo) y el sexto factor se llamó $O$ tras Fortalezas Asociadas (agrupando fortalezas como la clemencia, la curiosidad, la esperanza y la vitalidad).

$\mathrm{Si}$ bien estas agrupaciones de fortalezas no coincidieron con la clasificación oficial de seis virtudes y 24 fortalezas de Peterson y Seligman (2004), no se puede dejar de mencionar que la clasificación VIA se generó a partir de un seguimiento teórico de las tradiciones filosóficas y religiosas de Oriente y Occidente, y no como resultado de un análisis factorial. Asimismo, estudios previos tampoco pudieron replicar la clasificación VIA al realizar los análisis exploratorios de sus instrumentos (Brdar \& Kashdan, 2010; Cosentino, 2010; Giménez, 2010; Littman-Ovadia \& Lavy, 2012; Park \& Peterson, 2006b). No obstante esto, los autores no reniegan de estas inconsistencias debido a que desde un principio, la clasificación VIA se construyó en carácter tentativo para que con los avances en el estudio científico de la excelencia moral, pueda ser revisada e incluso alterada.

Cabe destacar que los expertos en el estudio de las fortalezas del carácter (Macdonald et al., 2008) afirman que estas diferencias halladas en la estructuración de los datos de ninguna manera invalidan los instrumentos construidos hasta el momento sino que, por el contrario, se los continúa utilizando en una gran cantidad de investigaciones ya que permiten, a diferencia de los instrumentos que evalúan las fortalezas de forma individual, superar el estudio fragmentario del carácter.

En cuanto a evidencias de validez convergente, se encontraron, conforme a lo esperado, correlaciones estadísticamente significativas y positivas entre las puntuaciones arrojadas por el IVyF Niños y la escala de deseabilidad social EDESI, el cuestionario de personalidad CAPI y la pregunta sobre satisfacción con la vida "¿Cuán satisfecho o contento te encuentras con tu vida?".

En este sentido, se encontraron correlaciones estadísticamente significativas y directas entre la satisfacción con la vida y todas las fortalezas de carácter exceptuando creatividad, valentía y perspectiva. Dos de las asociaciones más elevadas se obtuvieron con las fortalezas vitalidad y esperanza. Estos resultados son coincidentes con estudios previos llevados a cabo tanto con adultos (Cosentino, 2010; Park, Peterson \& Seligman, 2004; 2006) como con adolescentes (Giménez, 2010; Park \& Peterson, 2009) y niños (Park $\&$ Peterson, 2006a). 
Acerca de la deseabilidad social, si bien Peterson y Seligman (2004) no encontraron correlaciones significativas entre las fortalezas de carácter y el índice de deseabilidad social de Marlowe-Crowne (a excepción de prudencia y espiritualidad), en el presente estudio 14 de las 24 fortalezas se asociaron de forma estadísticamente significativa con este constructo. Esto coincide con los resultados encontrados en las investigaciones de Macdonald, Bore y Munro (2008), Osin (2009) y Ruch et al. (2010), en las cuales se halló que más de la mitad de las fortalezas correlacionaban con las puntuaciones de una escala que evaluaba deseabilidad social.

Sobre la asociación existente entre la personalidad y las fortalezas del carácter, Peterson y Seligman (2004) han hipotetizado que los factores del Big Five que se corresponden con las fortalezas del carácter de la clasificación VIA son: a) Responsabilidad con autorregulación y persistencia, b) Apertura a la Experiencia con curiosidad, apreciación de la belleza y creatividad y c) Extraversión con vitalidad y humor.

De las hipótesis anteriormente mencionadas, debe decirse que muchas de ellas se han replicado en la presente investigación. Por ejemplo, Escrupulosidad (equivalente a Responsabilidad en adultos) registró correlaciones moderadas y significativas con las dos fortalezas hipotetizadas: persistencia y autorregulación. El factor Escrupulosidad hace referencia a tres facetas: el orden, la organización y la responsabilidad. Esto quiere decir que los niños que obtienen puntuaciones significativas en este factor, suelen encontrar placer en mantener las cosas en orden, organizar sus tareas y tener control sobre las actividades que realizan. A su vez, suelen ser persistentes, responsables y se esfuerzan mucho por conseguir sus objetivos, aunque para esto tengan que anteponer el deber al placer. Siguiendo esta línea, se considera esperable que estos mismos niños alberguen en su carácter las fortalezas perseverancia y autorregulación. Éstas tienen que ver con poner mucho esfuerzo para lograr una meta propuesta a pesar de los obstáculos que pueda representar esa tarea y poder regular, controlar o gobernar las propias respuestas -ya sean impulsos, pensamientos, acciones o emocionesen pos del cumplimiento de ese objetivo (Peterson \& Seligman, 2004; Peterson \& Park, 2009).

A su vez, el factor Apertura a la Experiencia presentó asociaciones (aunque bajas) con dos de las fortalezas hipotetizadas: apreciación de la belleza y curiosidad. Puntuaciones altas en el factor Apertura a la Experiencia habla de niños que muestran preferencia por vivir nuevas experiencias, que gustan de explorar lo desconocido, que son curiosos del mundo exterior y proclives a innovar y generar ideas nuevas. En este sentido, se considera esperable que este factor se haya asociado con fortalezas tales como la apreciación por la belleza y la curiosidad, las cuales tienen que ver con disfrutar y encontrar cosas que aprender y conocer en cualquier aspecto de la vida, por más simple que ésta sea: la naturaleza, el arte, incluso las matemáticas o cualquier experiencia diaria (Peterson \& Seligman, 2004). Sin embargo, llama la atención que no se hayan encontrado asociaciones significativas entre Apertura a la Experiencia y creatividad, siendo que una de las facetas de este factor (denominada innovación) hace referencia a la capacidad de crear y de salir de lo cotidiano.

El factor Mesura no registró correlaciones con las dos fortalezas hipotetizadas. Sin embargo, se hallaron correlaciones moderadamente significativas con las fortalezas clemencia, prudencia y autorregulación. Los niños con puntuaciones altas en el factor Mesura, suelen presentar una conducta más tranquila y adaptada desde el punto de vista social. Una de las facetas que integran este factor es la adaptabilidad, la cual hace referencia a la franqueza, la sinceridad, a la tendencia a perdonar y olvidar. Los niños que puntúan alto se describen como cooperativos, respetuosos y honestos. Así, aunque no se hayan encontrado coincidencias con lo hipotetizado por Peterson y Seligman (2004), resulta esperable que este factor correlacione con las fortalezas clemencia, autorregulación y prudencia.

Por su parte, el factor Extraversión no presentó ninguna de las correlaciones hipotetizadas. No obstante, registró asociaciones bajas con las fortalezas apreciación de la belleza, inteligencia social, bondad y esperanza. El factor Extraversión presenta dos facetas: sentimiento gregario y emociones positivas por un lado y confianza por el otro. La primera faceta tiene que ver con niños que prefieren estar en compañía, en interacción social, con tendencia a experimentar más frecuentemente emociones de alegría. Se trata de niños que disfrutan intensamente lo que hacen, entreteniéndose fácilmente; de buen humor y optimistas. Las correlaciones halladas con las fortalezas apreciación de la belleza, inteligencia social y esperanza encontrarían su explicación en esta primera faceta. Por otro lado, la faceta confianza implica la tendencia a atribuir intenciones benévolas a los demás. Los niños que obtienen puntajes altos en esta faceta creen que los otros son buenos, sinceros, honestos y tienen buenas intenciones. En este sentido, la asociación significativa encontrada entre el factor Extraversión y la fortaleza bondad, encontraría su argumento en esta última faceta. 
Por otra parte, se analizaron las diferencias en las fortalezas del carácter de acuerdo al género. Los resultados han mostrado que las mujeres puntuaron más alto que los varones en las fortalezas amor y bondad. Estos resultados se encuentran en consonancia con estudios previos (Brdar, Anic \& Rijavec, 2011; Giménez, 2010; Park \& Peterson, 2003; Peterson \& Seligman, 2004; Shimai, Otake, Park, Peterson \& Seligman, 2006; Ruch, Weber, Park \& Peterson, 2014).

Como limitaciones de la presente investigación, se puede mencionar que al ser un instrumento de autopuntuación global directa, autores como Helms et al. (2006), mencionan que existe una dificultad en calcular la consistencia interna mediante el cálculo del alfa de Cronbach o la división por mitades, debido a que cada variable es evaluada por un único ítem. En este sentido, para futuras investigaciones se recomienda llevar a cabo un test re test que permita conocer la estabilidad de las puntuaciones en el tiempo.

Otra de las limitaciones del presente estudio consiste en la elección de la muestra, la cual está conformada por instituciones de clase económica media. Sería oportuno, en futuras investigaciones, contar con una muestra más heterogénea donde también participen instituciones educativas de clase alta o clase baja. Otra de las limitaciones encontradas tiene que ver con el formato de evaluación de los dos instrumentos utilizados, los cuales son autoinformes. Sería acertado para futuros estudios contar con otras fuentes de información.

En conclusión, tal como se mencionó en el marco teórico, los instrumentos para evaluar fortalezas del carácter en niños son pocos $\mathrm{y}$, particularmente en Argentina, no existe aún un instrumento para evaluar las 24 fortalezas de acuerdo a la clasificación VIA en población infantil. En respuesta a esto, el principal aporte de la presente investigación consistió en la adaptación y validación del IVyF Niños. De acuerdo a los resultados comentados, el IVyF Niños es un instrumento de rápida y sencilla administración, adecuado para la evaluación de las fortalezas humanas según la clasificación del carácter de Peterson y Seligman (2004).

\section{Referencias}

Anastasi, A. \& Urbina, S. (1998). Tests Psicológicos. México: Prentice Hall.

Brdar, I. \& Kashdan, T. B. (2010). Character strengths and well-being in Croatia: An empirical investigation of structure and correlates. Journal of research in personality, 44, 151-154. doi:10.1016/j. jrp.2009.12.001

Brdar, I., Anić, P. \& Rijavec, M. (2011). Character Strengths and Well-Being: Are There Gender Differences?. En I. Brdar (Ed.),
The human pursuit of well-being: a cultural approach (pp. 145156). Netherlands: Springer.

Byrne, B. M. (2009). Structural equation modeling with AMOS. New York: Routledge.

Cillessen, A. \& Rose, A. (2005). Understanding popularity in the peer system. Current Directions in Psychoogical Science, 14, 102-105. doi:10.1111/j.0963-7214.2005.00343.x

Cosentino, A. C. (2009). Evaluación de las Virtudes y Fortalezas Humanas en Población de Habla Hispana. Psicodebate: Psicología, Cultura y Sociedad, 10, 53-71. Recuperado de http://www.palermo.edu/cienciassociales/publicaciones/pdf/psico10/10Psico_03. pdf

Cosentino, A. C. (2010). Fortalezas del carácter en militares argentinos (Tesis doctoral). Universidad de Palermo, Argentina.

Cosentino, A. C. \& Castro Solano, A. (2008). Inventario de virtudes y fortalezas. Manuscrito no publicado.

Costa, P. T. \& McCrae, R. R. (1980). Influence of extraversion and neuroticism on subjective wellbeing: Happy and unhappy people. Journal of Personality and Social Psychology, 38(4), 668-678. doi:10.1037/0022-3514.38.4.668

Costa, P. T. \& McCrae, R. R. (1991). NEO PI-R. Professional manual. Revised NEO personality inventory (NEO PI-R) and NEO five-factor inventory (NEO-FFI). USA: Psychological Assesment Resources, Inc.

Costa, P. T., McCrae, R. R. \& Martin, T. A. (2008). Incipient adult personality: The NEO-PI-3 in middle-school-aged children. British Journal of Developmental Psychology, 26(1), 71-89. doi:10.1348/026151007X196273

Dahlsgaard, K., Peterson, C. \& Seligman, M. (2005). Shared virtue: The convergence of valued human strengths across culture and history. Review of General Psychology, 9, 203-213. doi:10.1037/1089-2680.9.3.203

Erickson, S. J. \& Feldstein, S. W. (2007). Adolescent Humor and its relationship Coping, Defense Strategies, Psychological Distress, and Well Being. Child Psychiatry and Human Development, 37, 255-271. doi:10.1007/s10578-006-0034-5

Ferragut, M., Blanca, M. J. \& Ortiz-Tallo, M. (2014). Psychological virtues during adolescence: A longitudinal study of gender differences. European Journal of Developmental Psychology, 11(5), 1-11. doi:10.1080/17405629.2013.876403

Froh, J. J., Yurkewicz, C. \& Kashdan, T. B. (2009). Gratitude and subjective well-being in early adolescence: Examining gender differences. Journal of adolescence, 32(3), 633-650. doi:10.1016/j. adolescence.2008.06.006

Giménez, M. (2010). La medida de las fortalezas psicológicas en adolescentes (VIAY): relación con clima familiar, psicopatología y bienestar psicológico (Tesis doctoral inédita). Universidad Complutense, Madrid.

Hair, J. F., Anderson, R. E., Tatham, R. L. \& Black, W. C. (1999). Análisis multivariante. Madrid: Prentice Hall.

Helms, J. E., Henze, K. T., Sass, T. L. \& Mifsud, V. A. (2006). Treating Cronbach's alpha reliability coefficients as data in counseling research. The counseling Psychologist, 34(5), 630-660. doi: $10.1177 / 0011000006288308$

Kashdan, T. B. \& Yuen, M. (2007). Wether highly curious students thrive academically depends on perceptions about the school learning environment: a study of Hong Kong adolescents. Motivation and Emotion, 31(4), 260-270. doi:10.1007/s11031-007-9074-9

Lampropoulos, G. K. (2001). Integrating Psychopathology, Positive Psychology and Psychotherapy. American Psychologist, 56(1), 87-88. doi:10.1037/0003-066X.56.1.87

Lemos, V. (2004). Operacionalización del constructo personalidad infantil a partir del enfoque de los Cinco Grandes Factores de 
Personalidad (Tesis doctoral). Universidad Nacional de San Luis, Argentina.

Lemos, V. (2005). Construcción y validación de una Escala para la Evaluación de la Deseabilidad Social Infantil (EDESI). Interdisciplinaria, 22(1), 79-96. Recuperado de http://www.scielo.org.ar/ scielo.php?script=sci_arttext\&pid=S1668-70272005000100004

Lemos, V. (2006). La evaluación de la personalidad infantil a partir del enfoque de los cinco grandes factores de personalidad (Big Five). Psicodiagnosticar, 16, 97-108. Recuperado de http://www. researchgate.net/publication/264541317_La_evaluacin_de_la_ personalidad_infantil_a_partir_del_enfoque_de_los_cinco_grandes_factores_de_personalidad_(Big_Five)

Linley, A., Joseph, A., Harrington, S. \& Wood, A. (2006). Positive psychology: Past, present and (possible) future. The Journal of Positive Psychology, 1(1), 3-16. doi:10.1080/17439760500372796

Littman-Ovadia, H. \& Lavy, S. (2012). Character Strengths in Israel. Hebrew adaptation of the VIA Inventory of Strengths. European Journal of Psychological Assessment, 28, 41-50.

Macdonald, C., Bore, M. \& Munro, D. (2008). Values in action scale and the Big 5: An empirical indication of structure. Journal of Research in Personality, 42(4), 787-799. doi:10.1027/10155759/a000089

McCrae, R. R., Costa, P. T., Martin, T. A., Oryol, V. E., Rukavishni- kov, A. A., Senin, I. G., ... \& Urbánek, T. (2004). Consensual validation of personality traits across cultures. Journal of Research in Personality, 38, 179-201. doi:10.1016/S0092-6566(03)00056-4

McCrae, R. R., Terracciano, A. \& 78 Members of the Personality Profiles of Cultures Project (2005). Universal features of personality traits from the observer's perspective: Data from 50 cultures. Journal of Personality and Social Psychology, 88(3), 547-561. doi:10.1037/0022-3514.88.3.547

Mikulic, I. M. \& Fernández, G. L. (2006). Técnicas y procesos de evaluación psicológica. Importancia de la evaluación psicológica de las fortalezas en niños y adolescentes. Anuario de Investigaciones, 13, 279-287. Recuperado de http://www.scielo.org.ar/ scielo.php?script=sci_arttext\&pid=S1851-16862006000100029

Osin, E. N. (2009). Social desirability in positive psychology: bias or desirable sociality? En T. Freire (Ed.), Understanding positive life: research and practice on positive psychology (pp. 421-442). Lisboa: Climepsi Editores.

Park, N. (2004). Character strengths and positive youth development. The Annals of the American Academy of Political and Social Science, 591(1), 40-54. doi:10.1177/0002716203260079

Park, N. \& Peterson, C. (2003). Assessment of character strengths among youth: The Values in Action Inventory of Strengths for Youth. Conferencia presentada en Indicators of Positive Development Conference, Washington, Estados Unidos.

Park, N. \& Peterson, C. (2006a). Character Strengths and happiness among young children: Content analysis of parental descriptions. Journal of Happiness Studies, 7(3), 323-341. doi:10.1007/s10902005-3648-6
Park, N. \& Peterson, C. (2006b). Moral competence and character strenghts among adolescents: the development and validation of the Values in Action Inventory of Strenghts for Youth. Journal of Adolescence, 29(6), 891-909. doi:10.1016/j.adolescence.2006.04.011

Park, N. \& Peterson, C. (2009). Achieving and sustaining a good life. Perspectives on Psychological Science, 4(4), 422-428. doi:10.1111/j.1745-6924.2009.01149.x

Park, N., Peterson, C. \& Seligman, M. (2004). Strengths of character and well- being. Journal of Social and Clinical Phychology, 23(5), 603-619. doi:10.1521/jscp.23.5.603.50748

Park, N., Peterson, C. \& Seligman, M. (2006). Character strengths in fifty-four nations and the fifty US states. Journal of Positive Psychology, 1, 118-129. doi:10.1080/17439760600619567

Peña, D. (2002). Análisis de Datos Multivariantes. Madrid: McGrawHill.

Peterson, C. \& Seligman, M. (2004). Character strengths and virtues: A classification and handbook. Washington, DC: American Psychological Association/New York: Oxford University Press.

Peterson, C., Park, N. \& Seligman, M. (2005). Orientations to happiness and life satisfaction: The full life versus the empty life. Journal of Happiness Studies, 6(1), 25-41. doi:10.1007/ s10902-004-1278-z

Piaget, J. (1932). The moral judgment of the child (Trad. M. Worden) New York: Harcourt, Brace, and World.

Ruch, W., Proyer, R. T., Harzer, C., Park, N., Peterson, C. \& Seligman, M. (2010). Values in Action Inventory of Strenghts (VIA-IS): Adaptation and validation of the German version and the development of a peer-rating form. Journal of individual differences, 31, 138-149. doi:10.1027/1614-0001/a000022

Ruch, W., Weber, M., Park, N. \& Peterson, C. (2014). Character strengths in children and adolescents: Reliability and initial validity of the German Values in Action Inventory of Strengths for Youth (German VIA-Youth). European Journal of Psychological Assessment, 30(1), 57-64. Recuperado de http://dx.doi. org/10.1027/1015-5759/a000169

Seligman, M. (2002). Authentic happiness: Using the new positive psychology to realize your potential for lasting fulfilment. New York: Free Press.

Seligman, M. \& Csikszentmihalyi, M. (2000). Positive psychology: An introduction. American Psychologist, 55, 5-14. Recuperado de http://dx.doi.org/10.1037/0003-066X.55.1.5

Seligman, M, Reivich, K., Jaycox, L. \& Gillham, J. (1995). The optimistic child. New York: Houghton, Mifflin.

Shimai, S., Otake, K., Park, N., Peterson, C. \& Seligman, M. (2006). Convergence of character strengths in American and Japanese Young Adults. Journal of Happiness Studies, 7, 311-322. doi:10.1007/s10902-005-3647-7

Terjesen, M. D. Jacofsky, M., Froh, J \& DiGiuseppe, R. (2004). Integrating positive psychology into schools: Implications for practice. Psychology in the Schools, 41(1), 163-172. doi:10.1002/ pits. 10148 